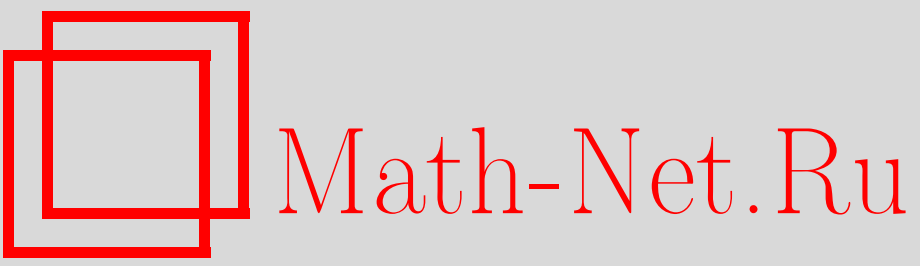

А. И. Алексеев, Об инфракрасном поведении глюонного пропагатора, ТМФ, 1996, том 106, номер 2, 250-263

DOI: https://doi.org/10.4213/tmf1111

Использование Общероссийского математического портала Math-Net.Ru подразумевает, что вы прочитали и согласны с пользовательским соглашением

http://www.mathnet.ru/rus/agreement

Параметры загрузки:

IP : 35.173 .137 .237

26 апреля 2023 г., 15:36:16 
ТЕОРЕТИЧЕСКАЯ

И МАТЕМАТИЧЕСКАЯ

ФИЗИКА

Том 106, № 2

февраль, 1996

А.И. Алексеев

\title{
ОБ ИНФРАКРАСНОМ ПОВЕДЕНИИ ГЛЮОННОГО ПРОПАГАТОРА
}

\begin{abstract}
В рамках глюодинамики рассматривается известное транкированное уравнение Швингера-Дайсона для глюонного пропагатора в аксиальной калибровке. Исследован общий случай степенного инфракрасного поведения с нецелыми показателями степени. Развита техника выделения нелидируюших членов нелинейного интегрального уравнения, определяемых только инфракрасным поведением пропагатора. Получено характеристическое уравнение на показатель степени и исследована область его значений $-1 \leq c \leq 3$. Показано, что при нецелых и неполуцелых значениях показателя степени рассматриваемое уравнение для глюонного пропагатора не имеет решений.
\end{abstract}

\section{1. ВВЕДЕНИЕ}

Выяснение поведения глюонного пропагатора в инфракрасной области является одной из важнейших задач для понимания непертурбативной области квантовой хромодинамики (КХД). В последнее время обсуждается возможность [1] слабосингулярного степенного поведения полного глюонного пропагатора в нуле. Под слабосингулярным при этом понимают поведение более мягкое, чем полюс у свободного пропагатора.

Интерес к слабосингулярному инфракрасному поведению связан с успехом [2] феноменологических моделей непертурбативного обмена кварками и глюонами при описании дифракционного рассеяния, глюонных структурных функций, упругого рассеяния, полных сечений. Слабосингулярное поведение глюонного пропагатора, с одной стороны, обеспечивает выполнение калибровочного условия $\pi_{\mu \nu}(0)=0$, а с другой стороны, позволяет в феноменологических приложениях считать поведение глюонного пропагатора в нуле практически константой.

В работах $[3,4]$ так же исследовалась возможность степенного инфракрасного поведения глюонного пропагатора, причем основной интерес был связан с поиском сингулярных решений конфайнментного типа. Под этим понималось инфракрасное поведение глюонного пропагатора, соответствующее росту кварк-антикваркового потенциала на больших расстояниях в приближении обмена одним одетым глюоном.

В качестве метода непертурбативного нерешеточного исследования инфракрасного поведения глюонных функций Грина и прежде всего пропагатора обычно используют точные уравнения для функций Грина - уравнения Швингера-Дайсона. Принципиальная трудность состоит в том, что цепочка этих уравнений является незамкнутой, и поэтому возникает задача обоснования метода транкирования. Сложность этой задачи 
заставляет привлекать физические соображения в пользу получаемых решений. Одним из методов, позволяющих в известной степени замкнуть систему уравнений, является использование $[5,6]$ следствий калибровочной инвариантности теории - тождеств Уорда-Славнова-Тейлора. При этом простота калибровочных тождеств делает привлекательным выбор аксиальной калибровки. Результаты проведенных исследований не позволяют сделать определенный вывод о поведении глюонного пропагатора в нуле. В ряде работ [5, 7-9] получено поведение глюонного пропагатора $D(q) \sim\left(q^{2}\right)^{-2}$, $q^{2} \rightarrow 0$. Это поведение дает линейньй кварк-антикварковый потенциал на больших расстояниях в приближении одноглюонного обмена. Кроме того, такое поведение служит основой при построении физически привлекательной картины вакуума КХД как дуальной сверхпроводяшей среды [10]. Обоснованию константного поведения глюонного пропагатора в нуле посвяшены работы [11]. Поведение $D(q) \sim 1 / q^{2} \ln ^{2}\left(q^{2} / \mu^{2}\right), q^{2} \rightarrow 0$, получено в работе [6] с использованием калибровочной техники.

Как отмечено в работах [1], неединственность решений может быть связана с нелинейностью соответствуюших интегральных уравнений. В более широком смысле принципиальным является вопрос, связана ли неединственность инфракрасного поведения глюонного пропагатора с различием методов транкирования уравнений для функций Грина или же она отражает сложную структуру вакуума КХД. Как показано в работах [12], при построении непертурбативных решений уравнений Швингера для производящего функционала и уравнений Швингера-Дайсона для функций Грина ключевой является проблема дополнительных граничных условий.

В данной работе мы рассмотрим уравнение, из исследования которого авторами работ [1] был сделан вывод о существовании решения для глюонного пропагатора со степенным слабосингулярным поведением в нуле, и покажем, что этот вывод не является обоснованным. Во втором разделе мы кратко обозначим метод получения основного интегрального уравнения, в третьем разделе мы исследуем инфракрасное поведение отдельных членов уравнения и получим характеристическую функцию для степенного инфракрасного поведения пропагатора в нуле. В четвертом разделе будет исследовано характеристическое уравнение и приведены результаты численного исследования характеристической функции. В заключении будут обсуждены полученные результаты и проведено сравнение с результатами работ [3, 4], а в приложении приведены формулы для инфракрасных асимптотик интегралов.

\section{2. ИНТЕГРАЛЬНОЕ УРАВНЕНИЕ ДЛЯ ГЛЮОННОГО ПРОПАГАТОРА}

Кратко опишем метод получения замкнутого интегрального уравнения [5] для глюонного пропагатора. Будем рассматривать теорию поля Янга-Миллса в бездуховой аксиальной калибровке [13] $A_{\mu}^{a} \eta_{\mu}=0, \eta_{\mu}$ - калибровочный вектор. Уравнение Швингера-Дайсона для глюонного пропагатора выглядит следующим образом:

$$
\begin{gathered}
P_{\mu \nu}(q)-P_{\mu \nu}^{(0)}(q)=\frac{1}{2} \int d k i \Gamma_{\mu \lambda \rho}^{(0)}\left(q,-k,-k^{\prime}\right) D_{\lambda \sigma}(k) \times \\
\times D_{\rho \delta}\left(k^{\prime}\right) i \Gamma_{\sigma \delta \nu}\left(k, k^{\prime},-q\right)+(1 / 6) \Delta_{\mu \nu}(q)+\Phi_{\mu \nu} .
\end{gathered}
$$

В этом уравнении $k^{\prime}=q-k ; \Delta_{\mu \nu}(q)$ - двухпетлевой член с полной четырехглюонной вершиной; $\Phi_{\mu \nu}$ - головастиковый член; $\Gamma_{\sigma \delta \nu}\left(k, k^{\prime},-q\right)$ - одночастично-неприводимая полная трехглюонная вершинная функция; $\Gamma_{\mu \lambda \rho}^{(0)}\left(q,-k,-k^{\prime}\right)-$ свободная трехглюонная 
вершинная функция, $d k=i g^{2} C_{2} d^{4} k /(2 \pi)^{4}$. Цветовые индексы здесь явно не выписаны, их легко восстановить, $C_{2}$ для цветовой группы $S U(N)$ равно $N$. Полный пропагатор $D_{\mu \nu}(q)$ с обратным пропагатором $P_{\mu \nu}(q)$ связан соотношением [5]

$$
D_{\mu \lambda}(q) P_{\lambda \nu}(q)=g_{\mu \nu}-q_{\mu} \eta_{\nu} /(q \eta)
$$

В аксиальной калибровке пропагатор, вообще говоря, зависит от двух скалярных функций. Следуя работам [5], сделаем предположение о пропорциональности полного пропагатора свободному:

$$
D_{\mu \nu}(q)=Z\left(-q^{2}\right) D_{\mu \nu}^{(0)}(q)=-\frac{Z\left(-q^{2}\right)}{q^{2}}\left[g_{\mu \nu}-\frac{q_{\mu} \eta_{\nu}+q_{\nu} \eta_{\mu}}{(q \eta)}+\frac{q_{\mu} q_{\nu} \eta^{2}}{(q \eta)^{2}}\right] .
$$

Тогда обратный пропагатор также характеризуется одной скалярной функцией:

$$
P_{\mu \nu}(q)=-\frac{q^{2}}{Z\left(-q^{2}\right)}\left(g_{\mu \nu}-\frac{q_{\mu} q_{\nu}}{q^{2}}\right)
$$

причем функция $Z$ зависит только от квадрата импульса. Тождество $\eta_{\mu} D_{\mu \nu}(k)=0$ позволяет от уравнения (1) перейти к свернутому с вектором $\eta_{\mu}$ уравнению, не содержашему двухпетлевых членов. Важным предположением работ [5] является предположение о доминантности продольной части вершинной функции при исследовании инфракрасного поведения глюонного пропагатора. Посредством калибровочного тождества Уорда-Славнова-Тейлора для трехглюонной вершины ее продольная часть может быть выражена [14] через скалярные функции, характеризующие пропагатор. Головастиковый член не зависит от внешнего импульса, и для не зависящей от калибровочного параметра $y=(k \eta)^{2} / k^{2} \eta^{2}$ функции $Z$ интегрирование по угловым переменным обращает его в нуль. Перейдя в евклидово пространство, приближенно проинтегрировав по угловым переменным методом работы [15], сделав соответствующие вычитания и наложив на внешний импульс и калибровочный вектор условие ортогональности, получаем [1] уравнение для перенормированной функции $Z_{R}\left(q^{2}\right)=Z\left(q^{2}\right) / Z\left(\mu^{2}\right), \mu^{2}$ - точка нормировки. Это уравнение имеет вид

$$
\begin{aligned}
& \frac{1}{Z_{R}\left(q^{2}\right)}=1+\frac{3 \alpha_{s}\left(\mu^{2}\right)}{\pi}\left(T_{1}+\frac{T_{2}}{Z_{R}\left(q^{2}\right)}\right) \\
T_{1}= & \int_{0}^{q^{2}} F_{1}\left(q^{2}, y\right) d y-\int_{0}^{\mu^{2}} F_{1}\left(\mu^{2}, y\right) d y+\int_{q^{2}}^{\infty} F_{2}\left(q^{2}, y\right) d y-\int_{\mu^{2}}^{\infty} F_{2}\left(\mu^{2}, y\right) d y \\
T_{2}= & \int_{0}^{q^{2}} F_{4}\left(q^{2}, y\right) d y-\int_{0}^{\mu^{2}} F_{4}\left(\mu^{2}, y\right) d y+\int_{q^{2}}^{\infty} F_{5}\left(q^{2}, y\right) d y-\int_{\mu^{2}}^{\infty} F_{5}\left(\mu^{2}, y\right) d y,
\end{aligned}
$$

где

$$
\begin{gathered}
F_{1}(x, y)=Z_{R}(x+y)\left[\frac{5 y}{12 x^{2}}+\frac{2}{3 x}+\frac{2}{3\left(y+\mu^{2}\right)}-\frac{y^{2}}{12 x^{3}}\right]+ \\
\quad+Z_{2}(x, y)\left[\frac{y^{3}}{24 x^{3}}-\frac{y^{2}}{4 x^{2}}-\frac{y}{4 x}\right]+\frac{3}{4 x}\left[Z_{R}(y)+y \frac{d Z_{R}(y)}{d y}\right], \\
F_{2}(x, y)=\frac{2 Z_{R}(x+y)}{3\left(y+\mu^{2}\right)}-\frac{Z_{R}(x+y)}{4 y}-\left(\frac{3 y}{4 x}+\frac{1}{3}+\frac{x}{8 y}\right) Z_{2}(x, y)+\frac{3 y}{4 x} \frac{d Z_{R}(y)}{d y},
\end{gathered}
$$




$$
\begin{aligned}
F_{4}(x, y)= & {\left[-\frac{y}{6 x^{2}}-\frac{2}{3 x}-\frac{2}{3\left(y+\mu^{2}\right)}\right] Z_{R}(x+y) Z_{R}(y)-} \\
& \quad-Z_{R}(x+y) Z_{4}(y, x) \frac{2\left(x+\mu^{2}\right)}{3\left(y+\mu^{2}\right)} \\
F_{5}(x, y)= & Z_{R}(x+y) Z_{R}(y)\left[\frac{7}{6 y}-\frac{2}{3\left(y+\mu^{2}\right)}\right]-Z_{R}(x+y) Z_{4}(y, x) \frac{2\left(x+\mu^{2}\right)}{3\left(y+\mu^{2}\right)} .
\end{aligned}
$$

Функции $Z_{2}, Z_{4}$ в формулах (6) выражаются через функцию $Z_{R}$ следуюшим образом:

$$
Z_{2}(x, y)=\left(Z_{R}(x+y)-Z_{R}(y)\right) / x, \quad Z_{4}(x, y)=\left(Z_{R}(x)-Z_{R}(y)\right) /(x-y) .
$$

Заметим, что использованная схема вычитаний отличается от стандартной. Предполагается, что уравнение (5), полученное в результате описанных выше приближений, дает правильное инфракрасное поведение пропагатора и не претендует на точное описание ультрафиолетового поведения пропагатора. В результате численного исследования уравнения (5) в работах [1] было сделано утверждение о наличии решения со слабосингулярным степенным поведением функции $Z_{R}\left(q^{2}\right)$ в нуле. Интегрирование в уравнении (5) осушествляется по всем значениям $q^{2}$. В работах [1] рассматривались два варианта поведения пропагатора при $q^{2} \rightarrow \infty$, отличающихся от поведения свободного пропагатора на степень логарифма. Как оказалось, точное поведение при $q^{2} \rightarrow \infty$ не сильно влияет на поведение решения в нуле. Путем введения ряда числовых параметров было осушествлено гладкое сшивание функции $Z_{R}\left(q^{2}\right)$ с заданным поведением в нуле и на бесконечности. Фитирование параметров показало, что с достаточной точностью функция $Z_{R}\left(q^{2}\right)$ ведет себя как $\left(q^{2}\right)^{1-c}$ при $q^{2} \rightarrow 0$, причем значение параметра $c$ находится в интервале от 0,01 до 0,3 , а более сингулярное поведение пропагатора исключается. С большей точностью численно определить параметр $c$ не удалось.

Мы покажем, что для нецелых и неполуцелых значений показателя степени $c$ действительно поведение пропагатора при средних и больших значениях импульса не сушественно и получим характеристическое уравнение для определения параметра $c$.

\section{3. ХАРАКТЕРИСТИЧЕСКАЯ ФУНКЦИЯ ДЛЯ СТЕПЕННОГО ИНФРАКРАСНОГО ПОВЕДЕНИЯ ГЛЮОННОГО ПРОПАГАТОРА}

Предположим, что функция $Z_{R}\left(q^{2}\right)$ не имеет особенностей на действительной полуоси $0<q^{2}<\infty$, поведение функции $Z_{R}\left(q^{2}\right)$ на бесконечности адекватно вычитательной процедуре (например, степень логарифма $q^{2}$ ), а инфракрасное поведение может быть описано суперпозицией членов со степенным поведением в нуле вида

$$
Z_{R}^{-1}(x)=\sum_{n=1}^{N}\left(\frac{x}{\mu^{2}}\right)^{c_{n}} f_{n}\left(\frac{x}{\mu^{2}}\right)+f_{0}\left(\frac{x}{\mu^{2}}\right) .
$$

Функции $f_{k}\left(x / \mu^{2}\right), \quad k=0,1, \ldots, N$, будем считать голоморфными в нуле, а параметры $c_{n}$ - нецелыми числами, занумерованными в порядке возрастания, $c_{1}<c_{2}<\ldots<$ $c_{N}$. Мы рассматриваем возможность степенного инфракрасного поведения пропагатора, поэтому член в формуле $(7)$, ведущий себя как $x^{c_{1}}$, будем считать лидирующим при $x \rightarrow 0$ и соответственно считать $f_{1}(0) \neq 0$. Тогда при $x \rightarrow 0$

$$
Z_{R}(x)=\alpha_{0}\left(x / \mu^{2}\right)^{1-c}+o\left(\left(x / \mu^{2}\right)^{1-c}\right),
$$


где $c=c_{1}+1, \alpha_{0}=f_{1}^{-1}(0)$. Соответствуюшей перестройкой членов в формуле $(7)$ можно добиться того, чтобы среди параметров $c_{n}$ не было параметров, отличающихся друг от друга на целое число, так что будем считать, что $c_{i}+k \neq c_{j}, k=1,2, \ldots, i<j$.

Мы воспользуемся тем обстоятельством, что для степенного инфракрасного поведения с нецельм показателем степени мы можем извлечь из интегралов, входяших в величины $T_{1}$ и $T_{2}$, члены, степенным образом зависяшие от $q^{2}$ при $q^{2} \rightarrow 0$ для любого неособенного поведения функции $Z_{R}(x)$ при конечных положительных значениях $x$.

Интегралы по $y$ от нуля до $q^{2}$ будем обозначать как $I$, а интегралы по $y$ от $q^{2}$ до бесконечности будем обозначать через $J$ с различными индексами. Имея дело с отдельными интегралами, мы будем вводить вместо бесконечного предела интегрирования вспомогательную константу $\Lambda_{\infty}^{2}$, которая не войдет в окончательные выражения для величин $T_{1}$ и $T_{2}$, не содержащих ультрафиолетовых расходимостей. Мы можем записать следуюшие выражения для $T_{1}$ и $T_{2}$ через интегралы типов $I$ и $J$ :

$$
\begin{aligned}
T_{1}=\frac{3}{4} & I_{1}^{0}+\frac{1}{4} I_{1}^{1}+\frac{1}{4} I_{1}^{2}-\frac{1}{24} I_{1}^{3}+\frac{2}{3} I_{2}^{0}+\frac{1}{6} I_{2}^{1}-\frac{1}{3} I_{2}^{2}+\frac{1}{24} I_{2}^{3}+ \\
& +\frac{2}{3} \frac{q^{2}}{\mu^{2}} I_{3}+\frac{1}{8} J_{1}^{-1}+\frac{1}{3} J_{1}^{0}+\frac{3}{4} J_{1}^{1}-\frac{3}{8} J_{2}^{-1}-\frac{1}{3} J_{2}^{0}-\frac{3}{4} J_{2}^{1}+ \\
& +\frac{2}{3} \frac{q^{2}}{\mu^{2}} J_{3}+\frac{3}{4} \frac{A_{\infty}}{q^{2}}-\int_{0}^{\mu^{2}} F_{1}\left(\mu^{2}, y\right) d y-\int_{\mu^{2}}^{\Lambda_{\infty}^{2}} F_{2}\left(\mu^{2}, y\right) d y \\
T_{2}=- & \frac{2}{3} I_{4}^{0}-\frac{1}{6} I_{4}^{1}-\frac{2}{3} \frac{q^{2}}{\mu^{2}} I_{5}-\frac{2}{3} \frac{\left(q^{2}+\mu^{2}\right)}{\mu^{2}} I_{6}+\frac{7}{6} J_{4}^{-1}- \\
& -\frac{2}{3} \frac{q^{2}}{\mu^{2}} J_{5}-\frac{2}{3} \frac{\left(q^{2}+\mu^{2}\right)}{\mu^{2}} J_{6}-\int_{0}^{\mu^{2}} F_{4}\left(\mu^{2}, y\right) d y-\int_{\mu^{2}}^{\Lambda_{\infty}^{2}} F_{5}\left(\mu^{2}, y\right) d y .
\end{aligned}
$$

Входящие в формулы $(9),(10)$ интегралы типа $I$ определены следуюшим образом:

$$
\begin{aligned}
I_{1}^{k} & =\frac{1}{q^{2}} \int_{0}^{q^{2}} d y Z_{R}(y)\left(\frac{y}{q^{2}}\right)^{k}, \quad I_{2}^{k}=\frac{1}{q^{2}} \int_{0}^{q^{2}} d y Z_{R}\left(q^{2}+y\right)\left(\frac{y}{q^{2}}\right)^{k}, \\
I_{3} & =\frac{\mu^{2}}{q^{2}} \int_{0}^{q^{2}} d y \frac{Z_{R}\left(q^{2}+y\right)}{y+\mu^{2}}, \quad I_{4}^{k}=\frac{1}{q^{2}} \int_{0}^{q^{2}} d y Z_{R}(y) Z_{R}\left(q^{2}+y\right)\left(\frac{y}{q^{2}}\right)^{k}, \\
I_{5} & =\frac{\mu^{2}}{q^{2}} \int_{0}^{q^{2}} d y Z_{R}(y) Z_{R}\left(q^{2}+y\right) \frac{1}{y+\mu^{2}}, \\
I_{6} & =\mu^{2} \int_{0}^{q^{2}} d y Z_{R}\left(q^{2}+y\right) \frac{Z_{R}\left(q^{2}\right)-Z_{R}(y)}{\left(q^{2}-y\right)\left(y+\mu^{2}\right)} .
\end{aligned}
$$

Интегралы типа $J$ в формулах $(9),(10)$ определены подобным же образом, только интегрирование по $y$ ведется от $q^{2}$ до $\Lambda_{\infty}^{2}$. Константа $A_{\infty}$ в формуле $(9)$ имеет вид

$$
A_{\infty}=\int_{0}^{\Lambda_{\infty}^{2}} y \frac{d Z_{R}(y)}{d y} d y .
$$

Для степенного поведения $(8)$ при $q^{2} \rightarrow 0$ интегралы типа $I$ ведут себя следующим образом:

$$
\begin{aligned}
& I_{m}^{k}=\alpha_{0}\left(q^{2} / \mu^{2}\right)^{1-c} i_{m}^{k}(c)+o\left(\left(q^{2} / \mu^{2}\right)^{1-c}\right), \quad m=1,2,3, \\
& I_{m}^{k}=\alpha_{0}^{2}\left(q^{2} / \mu^{2}\right)^{2-2 c} i_{m}^{k}(c)+o\left(\left(q^{2} / \mu^{2}\right)^{2-2 c}\right), \quad m=4,5,6 .
\end{aligned}
$$


Анализ интегралов типа $J$ показывает, что при $q^{2} \rightarrow 0$, помимо степенных членов, в них имеются ряды по целым степеням $q^{2}$, а также члены, ведущие себя как $1 /\left(q^{2}\right)^{m}$. В приложении вычислены члены интегралов типов $I$ и $J$, фигурирующие в формулах (9), $(10)$ и ведушие себя при $q^{2} \rightarrow 0$ как $\left(q^{2}\right)^{1-c},\left(q^{2}\right)^{2-2 c}, 1 / q^{2}, 1 /\left(q^{2}\right)^{2}$. Подставим в формулы $(9),(10)$ выражения для интегралов типов $I$ и $J$ из приложения. При этом члены, ведущие себя как $1 / q^{2}, 1 /\left(q^{2}\right)^{2}$, взаимно сокращаются. Воспользовавшись рекуррентными соотношениями для $i_{2}^{k}(c)$ и вводя регулярную функцию $\gamma(x)$ :

$$
\gamma(x)=\left(2^{x}-1\right) / x
$$

при $q^{2} \rightarrow 0$ получаем

$$
\begin{aligned}
& T_{1}= P_{1}\left(q^{2}\right)+\alpha_{0}\left(q^{2} / \mu^{2}\right)^{-c_{1}} \Delta_{1}(c)+o\left(\left(q^{2} / \mu^{2}\right)^{-c_{1}}\right) \\
& \Delta_{1}(c)=\frac{1}{24} {\left[-\frac{1}{5-c}+\frac{6}{4-c}+\frac{6}{3-c}-\frac{3}{1-c}-9 j_{2}^{-1}(c)+\gamma(5-c)-\right.} \\
&\quad-11 \gamma(4-c)+41 \gamma(3-c)-7 \gamma(2-c)] ; \\
& T_{2}= P_{2}\left(q^{2}\right)+Z_{R}\left(q^{2}\right) Q_{2}\left(q^{2}\right)+\alpha_{0}^{2}\left(q^{2} / \mu^{2}\right)^{-2 c_{1}} \Delta_{2}(c)+o\left(\left(q^{2} / \mu^{2}\right)^{-2 c_{1}}\right) ; \\
& \Delta_{2}(c)=\frac{1}{6}\left[-4 i_{4}^{0}(c)-i_{4}^{1}(c)-4 i_{6}+7 j_{4}(c)-4 j_{6}(c)\right] .
\end{aligned}
$$

В формулах $(15),(17) \quad P_{1}\left(q^{2}\right), P_{2}\left(q^{2}\right), Q_{2}\left(q^{2}\right)$ - не определяемые нами функции, регулярные в точке $q^{2}=0$ и зависящие от поведения $Z_{R}\left(q^{2}\right)$ при всех значениях $q^{2}$. С учетом соотношений $x^{\alpha} o\left(x^{\beta}\right)=o\left(x^{\alpha+\beta}\right), o\left(x^{\alpha}\right) o\left(x^{\beta}\right)=o\left(x^{\alpha+\beta}\right), x \rightarrow 0$, уравнение (5) можно записать в виде

$$
\begin{aligned}
\frac{1}{Z_{R}\left(q^{2}\right)}=1 & +\frac{3 \alpha_{s}\left(\mu^{2}\right)}{\pi}\left\{P_{1}\left(q^{2}\right)+Q_{2}\left(q^{2}\right)+\frac{1}{Z_{R}\left(q^{2}\right)} P_{2}\left(q^{2}\right)+\right. \\
& +\alpha_{0}\left(\frac{q^{2}}{\mu^{2}}\right)^{-c_{1}} \Delta(c)+o\left(\left(q^{2} / \mu^{2}\right)^{\left.-c_{1}\right)}\right\},
\end{aligned}
$$

где $\Delta(c)=\Delta_{1}(c)+\Delta_{2}(c)$ - характеристическая функция. В соответствии с нашим предположением (7) о поведении функции $Z_{R}^{-1}(x)$ в нуле функции $f_{n}$, а также функции $P_{1}$, $P_{2}, Q_{2}$ можно разложить в нуле в степенные ряды с ненулевыми радиусами сходимости:

$$
\begin{aligned}
f_{n}\left(q^{2} / \mu^{2}\right) & =\sum_{i=0}^{\infty} f_{n}^{i}\left(q^{2} / \mu^{2}\right)^{i}, \quad P_{1,2}\left(q^{2}\right)=\sum_{i=0}^{\infty} p_{1,2}^{i}\left(q^{2} / \mu^{2}\right)^{i} \\
Q_{2}\left(q^{2}\right) & =\sum_{i=0}^{\infty} q_{2}^{i}\left(q^{2} / \mu^{2}\right)^{i} .
\end{aligned}
$$

Произведения степенных рядов мы можем записать так же в виде степенных рядов, и тогда мы получаем

$$
Z_{R}^{-1}\left(q^{2}\right) P_{2}\left(q^{2}\right)=\sum_{n=1}^{N}\left(q^{2} / \mu^{2}\right)^{c_{n}} A_{n}\left(q^{2} / \mu^{2}\right)+A_{0}\left(q^{2} / \mu^{2}\right)
$$


где голоморфные в нуле функции $A_{i}, i=0,1, \ldots, N$, могут быть представлены в виде

$$
A_{i}\left(q^{2} / \mu^{2}\right)=\sum_{j=0}^{\infty} a_{i}^{j}\left(q^{2} / \mu^{2}\right)^{j}, \quad a_{i}^{j}=\sum_{k=0}^{j} f_{i}^{k} p_{2}^{j-k} .
$$

Используя формулы (7), (21), от уравнения (19) можно перейти к уравнению

$$
\begin{aligned}
& \sum_{n=1}^{N}\left(\frac{q^{2}}{\mu^{2}}\right)^{c_{n}}\left[f_{n}\left(\frac{q^{2}}{\mu^{2}}\right)-\frac{3 \alpha_{s}\left(\mu^{2}\right)}{\pi} A_{n}\left(\frac{q^{2}}{\mu^{2}}\right)\right]+ \\
& \quad+f_{0}\left(\frac{q^{2}}{\mu^{2}}\right)-1-\frac{3 \alpha_{s}\left(\mu^{2}\right)}{\pi}\left[P_{1}\left(q^{2}\right)+Q_{2}\left(q^{2}\right)+A_{0}\left(\frac{q^{2}}{\mu^{2}}\right)\right]= \\
& \quad=\frac{3 \alpha_{s}\left(\mu^{2}\right)}{\pi} \alpha_{0} \Delta(c)\left(\frac{q^{2}}{\mu^{2}}\right)^{-c_{1}}+o\left(\left(\frac{q^{2}}{\mu^{2}}\right)^{-c_{1}}\right), \quad q^{2} \rightarrow 0 .
\end{aligned}
$$

Из уравнения (23) видно, что при $c_{1}>0$ член, ведущий себя как $\left(q^{2}\right)^{-c_{1}}$, является лидируюшим при $q^{2} \rightarrow 0$. Поэтому для определения показателя степени инфракрасной асимптотики имеем характеристическое уравнение $\Delta(c)=0$. Мы считаем при этом, что если $\left.f_{0}(x)\right|_{x \sim 0} \sim x^{m}$, то $c_{1}<m$.

Рассмотрим случай $c_{1}<0$. Теперь определяемый инфракрасной областью член $\left(q^{2}\right)^{-c_{1}}$ в уравнении (23) не является лидируюшим. Как мы увидим далее, при $c=$ $1-n / 2, n=0,1,2, \ldots$, характеристическая функция имеет полюсы. Эти случаи следует рассматривать отдельно, поэтому будем считать $c_{1}+n \neq-c_{1}, n$-целое число, исключив тем самым из рассмотрения полуцелые значения показателя степени инфракрасной асимптотики. Нас интересуют члены уравнения $(23)$, которые при $q^{2} \rightarrow 0$ главнее и такие же, как член $\left(q^{2}\right)^{-c_{1}}$. Как было сказано ранее, среди параметров $c_{k}$ нет таких, которые отличаются друг от друга на целое число. Поэтому возможна интерференция члена $\left(q^{2}\right)^{-c_{1}}$ только с одним из членов в формуле $(23)$. Пусть это член $\left(q^{2}\right)^{c_{i}+N_{i}}$, т.е. $c_{i}+N_{i}=-c_{1}$. В зависимости от значений $c_{n}, f_{n}^{i}$ число членов, которые следует учесть, будет различным. Эти члены ведут себя при $x \rightarrow 0$ как $x^{c_{1}}, x^{c_{1}+1}, \ldots, x^{c_{1}+N_{1}}, x^{c_{2}}$, $x^{c_{2}+1}, \ldots, x^{c_{2}+N_{2}}, \ldots, x^{c_{i}}, x^{c_{i}+1}, \ldots, x^{c_{i}+N_{i}}, \ldots, x^{c_{k}}, x^{c_{k}+1}, \ldots, x^{c_{k}+N_{k}}, x^{0}, x$, $\ldots, x^{N_{0}}$. При этом для $j=1,2, \ldots, k N_{j}=\left[-c_{1}-c_{j}\right], N_{0}=\left[-c_{1}\right]$, где квадратные скобки означают целую часть числа. Для целых чисел $N_{j}$ выполняется соотношение $N_{1} \geq N_{2} \geq \ldots \geq N_{i}>N_{i+1} \geq \ldots \geq N_{k} \geq 0$, а также $N_{0} \geq 0$. Расписанное по степеням $q^{2}$ уравнение (23) имеет следующую структуру:

$$
\sum_{m=1}^{M} b_{m} x^{\alpha_{m}}+o\left(x^{-c_{1}}\right)=0, \quad x \rightarrow 0
$$

где $M=\sum_{j=0}^{k} N_{j}+k+1$, а $\alpha_{m} \neq \alpha_{m^{\prime}}$ при $m^{\prime} \neq m$. Решая уравнение $(24)$ с точностью до $x^{-c_{1}}$, получаем $b_{m}=0, m=1,2, \ldots, M$. Тогда из формул $(20),(22),(23)$ получаем систему уравнений

$$
\begin{gathered}
f_{n}^{j}-\frac{3 \alpha_{s}\left(\mu^{2}\right)}{\pi} a_{n}^{j}=0, \quad n=1,2, \ldots, k, \quad j=0,1, \ldots, N_{n}, \quad(n-i)^{2}+\left(j-N_{i}\right)^{2} \neq 0 \\
f_{i}^{N_{i}}-\frac{3 \alpha_{s}\left(\mu^{2}\right)}{\pi}\left(a_{i}^{N_{i}}+\alpha_{0} \Delta(c)\right)=0
\end{gathered}
$$




$$
\begin{aligned}
& f_{0}^{0}-1-\frac{3 \alpha_{s}\left(\mu^{2}\right)}{\pi}\left(p_{1}^{0}+q_{2}^{0}+a_{0}^{0}\right)=0, \\
& f_{0}^{n}-\frac{3 \alpha_{s}\left(\mu^{2}\right)}{\pi}\left(p_{1}^{n}+q_{2}^{n}+a_{0}^{n}\right)=0, \quad n=1,2, \ldots, N_{0} .
\end{aligned}
$$

Решаем первое из уравнений системы (25) при $n=1, j=0,1, \ldots, N_{1}$. Используя формулы (22), получаем $p_{2}^{0}=\pi / 3 \alpha_{s}\left(\mu^{2}\right), p_{2}^{i}=0$ при $i=1,2, \ldots, N_{1}$. Тогда $a_{i}^{j}=f_{i}^{j} p_{2}^{0}=\pi\left(3 \alpha_{s}\left(\mu^{2}\right)\right)^{-1} f_{i}^{j}$ при $i=0,1, \ldots, k, j=0,1, \ldots, N_{1}$, и решая три последних уравнения системы $(25)$, окончательно получаем

$$
\begin{aligned}
p_{2}^{0} & =\pi / 3 \alpha_{s}\left(\mu^{2}\right), \quad p_{2}^{n}=0, \quad n=1,2, \ldots, N_{1}, \\
p_{1}^{0}+q_{2}^{0} & =-\pi / 3 \alpha_{s}\left(\mu^{2}\right), \quad p_{1}^{n}+q_{2}^{n}=0, \quad n=1,2, \ldots, N_{0}, \\
\Delta(c) & =0 .
\end{aligned}
$$

Здесь мы не будем рассматривать условия на поведение функции $Z_{R}\left(q^{2}\right)$ при промежуточных и больших значениях $q^{2}$, следующие из формул (26). Для нас существенно, что значение показателя степени инфракрасной асимптотики должно определяться характеристическим уравнением $\Delta(c)=0$. Это утверждение, естественно, остается в силе при отсутствии интерференции степенных членов в уравнении (23).

\section{4. ИССЛЕДОВАНИЕ ХАРАКТЕРИСТИЧЕСКОГО УРАВНЕНИЯ $\Delta(c)=0$}

Исследуем поведение характеристической функции $\Delta(c)$ при $-1 \leq c \leq 3$. При $c=-1,-1 / 2,0,1 / 2,1,3$ функция $\Delta(c)$ имеет простые полюсы. Мы выделим полюсные члены в явном виде, а для решения вопроса о корнях характеристического уравнения в промежуточных точках получим интегральное представление для $\Delta(c)$, удобное для численного исследования. Рассмотрим величины $\Delta_{1,2}(c)$. Сделаем в интегральном представлении (П.12) для $j_{2}^{-1}(c)$ три вычитания с тем, чтобы выделить полюсные члены при $c=-1,0,1$. С помошью рекуррентных соотношений (П.5) выразим $i_{4}^{0}(c)$ через $i_{4}^{1}(c)$ и воспользуемся интегральными представлениями для функций, входящих в выражение (18). В результате получаем

$$
\begin{aligned}
\Delta(c)=\frac{23}{24} & \frac{1}{c+1}-\frac{187}{96} \frac{1}{2 c+1}+\frac{7}{24} \frac{1}{c}-\frac{5}{12} \frac{1}{2 c-1}+ \\
& +\frac{2}{3} \frac{1}{c-1}-\frac{3}{4} \frac{1}{c-3}+\tilde{\Delta}(c) \\
\tilde{\Delta}(c)=\frac{1}{24} & \left\{\int _ { 0 } ^ { 1 } d t \left[( 1 + t ) ^ { 1 - c } \left(28 t^{-3+2 c}+28 t^{2-c}-9 t^{-2+c}-16\left(1+t^{-3+2 c}\right) \times\right.\right.\right. \\
& \left.\times\left(1-t^{1-c}\right) /(1-t)\right)-12 t^{-3+2 c}+4(1+3 c) t^{-2+2 c}+ \\
& +2\left(16-5 c-3 c^{2}\right) t^{-1+2 c}+2\left(c^{3}+4 c^{2}-13 c+16\right) t^{2 c}+ \\
& +(1 / 6)\left(192-122 c+51 c^{2}-22 c^{3}-3 c^{4}\right) t^{1+2 c}-16 t^{1-c}+ \\
& +4(4 c-15) t^{2-c}-7 t^{-2+c}+(7 c-23) t^{-1+c}-\left(7 c^{2}-\right. \\
& \left.-39 c+64) t^{c} / 2\right]-23 \gamma(2-c)+41 \gamma(3-c)-11 \gamma(4-c)+ \\
& \left.+\gamma(5-c)+\frac{6}{4-c}-\frac{1}{5-c}+63 / 4-17 c / 6+7 c^{2} / 12+c^{3} / 4\right\} .
\end{aligned}
$$




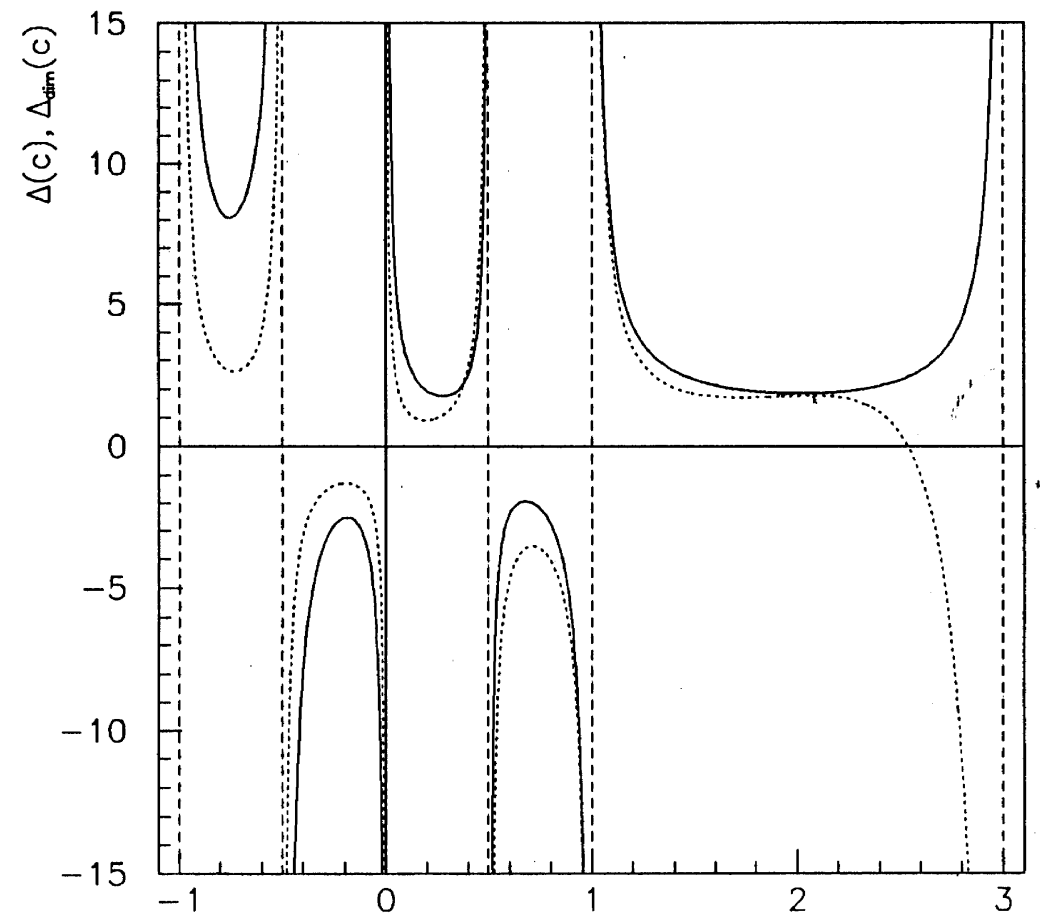

Характеристические функции для степенного инфракрасного поведения глюонного пропагатора: $\Delta(c)$ - для уравнения (5) (сплошная линия), $\Delta_{\operatorname{dim}}(c)$ - для уравнения (14) работы [3] (прерывистая линия).

Величина $\tilde{\Delta}(c)$ - регулярная часть $\Delta(c)$ в рассматриваемой области значений $c$. Вычитания в интегральном представлении для $\tilde{\Delta}(c)$ таковы, что при $-1 \leq c \leq 3$ подынтегральная функция конечна. Вычеты характеристической функции $\Delta(c)$ в полюсах $c=-1,-1 / 2,0,1 / 2,1,3$ имеют знаки,,,,,+-+-+- . Поэтому можно было бы ожидать, что у характеристического уравнения имеется четное количество корней на рассматриваемом отрезке $[-1,3]$ значений параметра $c$. На рисунке приведен график характеристической функции $\Delta(c)$, полученный в результате численного интегрирования. Видим, что характеристическое уравнение $\Delta(c)=0$ для степенного инфракрасного поведения глюонного пропагатора решений не имеет.

\section{5. ЗАКЛЮЧЕНИЕ}

Предположение о степенном инфракрасном поведении функции $Z_{R}^{-1}\left(q^{2}\right)$ вида (7) с нецелыми и неполуцелыми показателями степени позволило развить методику выделения степенных членов исследуемого интегрального уравнения (5), определяемых только инфракрасным поведением пропагатора. В уравнении (5) и соответственно в уравнении (19) это члены, имеюшие поведение $\left(q^{2}\right)^{-c_{1}}$ при $q^{2} \rightarrow 0$. На решении уравнение должно обращаться в тождество и, поскольку члены уравнения (24) имеют различное аналитическое поведение в нуле, то они должны обрашаться в нуль независимым об- 
разом. Эти условия, помимо не рассматриваемых нами условий на поведение $Z_{R}\left(q^{2}\right)$ при средних и больших $q^{2}$, дают характеристическое уравнение $\Delta(c)=0$ для определения показателя степени $c$. Мы показали, что при $-1 \leq c \leq 3$ это уравнение решений не имеет. При $c=c_{\min } \simeq 0,28$ характеристическая функция имеет локальный минимум $\Delta\left(c_{\min }\right) \simeq 1,76$, а при $c=c_{\max } \simeq 0,68$ она имеет локальный максимум $\Delta\left(c_{\max }\right) \simeq-1,93$. Слабая зависимость результатов численного анализа [1] от поведения функции $Z_{R}\left(q^{2}\right)$ при больших $q^{2}$ позволяет предположить, что лидируюшие при $q^{2} \rightarrow 0$ члены уравнения (24) численно малы. В этом случае фитирование параметров с целью минимизации модуля разности $Z_{R}^{\text {out }}\left(q^{2}\right)-Z_{R}^{\text {in }}\left(q^{2}\right)$ даст значение параметра $c$, соответствуюшее минимуму модуля характеристической функции $\Delta(c)$. Это позволяет объяснить результаты численного исследования работ [1].

Отметим, что в работе [16] рассмотрен частный случай степенного инфракрасного поведения $Z_{R}\left(q^{2}\right)=\left(q^{2}\right)^{-c_{1}} f\left(q^{2}\right)$ решения уравнения $(5)$, где $f(x)$ - регулярная в нуле функция, причем исследованная область значений параметра $-1<c_{1}<0$ отвечала слабосингулярному инфракрасному поведению. В работе [17] был исследован более широкий интервал значений $-2<c_{1}<2$ и решений характеристического уравнения также не было обнаружено.

Представляет интерес сравнить результаты настояшей работы с результатами предшествуюших работ по поиску решений полевых уравнений для пропагатора с дробным степенным инфракрасным поведением. В работе [3] получена характеристическая функция, выражающаяся через гамма-функции и гипергеометрические функции ${ }_{3} F_{2}$ от единичного аргумента, с параметрами, зависяшими от показателя степени инфракрасной асимптотики. При этом приближенное интегрирование по угловым переменным не производилось и использовалась размерная регуляризация, в которой ультрафиолетовые вычитания осуществляются автоматически. Характеристическую функцию работы [3], умноженную для перехода к используемой в настоящей работе нормировке на $-(2 \Gamma(c))^{-1}$, мы обозначим $\Delta_{\operatorname{dim}}(c)$. График этой функции приведен на рисунке. Мы видим, что при $c<2$ поведение функций $\Delta_{\operatorname{dim}}(c)$ и $\Delta(c)$ является близким, а при $c>2$ поведение функции $\Delta_{\operatorname{dim}}(c)$ качественно отличается от поведения функции $\Delta(c)$, и при $c \simeq 2,537$ она имеет корень, отвечающий почти осцилляторному поведению потенциала, $V(r) \sim r^{2,07}$. В работах [18] приведены аргументы в пользу осцилляторного поведения кварк-антикваркового потенциала на больших расстояниях. В работе [4] исследовалась инфракрасная асимптотика глюонного пропагатора с помощью уравнений без затравок для пропагаторов, и в приближении простых петель было получено уравнение самосогласования для нецелых показателей степени (индексов), определяющих аномальную размерность векторного поля. Заметим, что, помимо указанных в работе [4] решений $c \simeq 1,647, c \simeq 0,922, c \simeq 2,564$, уравнение имеет еше два корня: $c \simeq-0,498$ и $c \simeq-0,013$. Однако, как отмечается в работе [4], уравнение для индексов не дает ответа на вопрос, соответствуют ли его решения инфракрасной или ультрафиолетовой асимптотике пропагатора. Интересно отметить, что хотя в работе [4] использовалась лоренцева калибровка, один из корней весьма близок к корню характеристического уравнения работы [3].

Имея в виду проблему нахож дения самосогласованного решения для глюонного пропагатора в инфракрасной области, следовало бы более тшательно проанализировать приближения, приводящие к уравнению (5). Одной из возможностей является включение в рассмотрение поперечной части трехглюонной вершинной функции [8].

Автор глубоко признателен Б.А. Арбузову, В.Е. Рочеву, С.Н. Соколову за полезные обсуждения. Работа выполнена при поддержке Российского фонда фундаментальных 
исследований, грант № 95-02-03704-а.

\section{ПРИЛОЖКНИЕ}

Найдем поведение интегралов, входяших в формулы (9) и (10), при $q^{2} \rightarrow 0$ для степенного поведения функции $Z_{R}(x)$ в нуле. Рассмотрим интегралы типа $I$, определяемые формулами (11). Размерные факторы мы выделили согласно формулам (13). Непосредственная подстановка разложения (8) дает для интеграла $I_{1}^{k}$ следующий результат:

$$
i_{1}^{k}(c)=1 /(k+2-c) .
$$

Интеграл $I_{1}^{k}$ сходится при $c<k+2$. Для интеграла $I_{2}^{k}$ безразмерная величина $i_{2}^{k}(c)$ равна

$$
i_{2}^{k}(c)=\int_{0}^{1} d t t^{k}(1+t)^{1-c} .
$$

Интеграл $I_{2}^{k}$ сходится при любых значениях $c$. При анализе зависимости характеристической функции от параметра $c$ нам будет удобно пользоваться интегральными представлениями. Для обеспечения сходимости интегралов при $t=0$ в интересуюшей нас области значений параметра $c$ мы будем пользоваться аналитическими продолжениями интегралов, получаемыми вычитанием достаточного числа членов разложения подынтегральных выражений в нуле. Для вычисления интегралов (П.2) при различных $k$, $k=1,2,3, \ldots$, удобно воспользоваться рекуррентным соотношением

$$
i_{2}^{k}(c)=\frac{1}{2+k-c}\left(2^{2-c}-k i_{2}^{k-1}(c)\right)
$$

при этом

$$
i_{2}^{0}(c)=\frac{1}{2-c}\left(2^{2-c}-1\right) .
$$

Для интеграла $I_{3} \quad i_{3}(c)=i_{2}^{0}(c)$. Для интеграла $I_{4}^{k}$ получаем

$$
i_{4}^{k}=\int_{0}^{1} d t t^{k+1-c}(1+t)^{1-c} .
$$

Интеграл $I_{4}^{k}$ сходится при $c<k+2$. Для вычисления функции $i_{4}^{k}(c)$ при целых $k>0$ можно воспользоваться рекуррентной формулой

$$
i_{4}^{k}(c)=\frac{1}{3-2 c+k}\left[2^{2-c}-(1+k-c) i_{4}^{k-1}(c)\right] .
$$

Для интеграла $I_{5}$ имеем

$$
i_{5}(c)=i_{4}^{0}(c)
$$

Вычисляя интеграл $I_{6}$, получаем

$$
i_{6}(c)=\int_{0}^{1} d t(1+t)^{1-c} \frac{1-t^{1-c}}{1-t} .
$$

Интеграл $I_{6}$ и соответственно интеграл (П.7) сходятся при $c<2$. 
Перейдем к рассмотрению поведения интегралов типа $J$ при $q^{2} \rightarrow 0$. Рассмотрим интеграл $J_{1}^{k}$. При $c<2+k$ мы можем его представить как

$$
J_{1}^{k}=\frac{1}{\left(q^{2}\right)^{k+1}} \int_{0}^{\Lambda_{\infty}^{2}} d y y^{k} Z_{R}(y)-I_{1}^{k}
$$

где лидируюшим при $q^{2} \rightarrow 0$ является первый член. Обратимся к интегралу $J_{2}^{k}$ :

$$
J_{2}^{k}=\frac{1}{q^{2}} \int_{q^{2}}^{\Lambda_{\infty}^{2}} d y Z_{R}\left(q^{2}+y\right)\left(\frac{y}{q^{2}}\right)^{k}, \quad k=-1,0,1 .
$$

Пусть $k=-1$. Интеграл $J_{2}^{-1}$ представим в виде

$$
J_{2}^{-1}=\int_{q^{2}}^{\lambda^{2}} d y \frac{Z_{R}\left(q^{2}+y\right)}{y}+\int_{\lambda^{2}}^{\Lambda_{\infty}^{2}} d y \frac{Z_{R}\left(q^{2}+y\right)}{y} .
$$

Мы ввели такой достаточно малый параметр $\lambda^{2}$, что при $y<\lambda^{2}$ для подынтегральной функции $Z_{R}\left(q^{2}+y\right)$ можно воспользоваться асимптотической оценкой $(8)$. Область интегрирования $y>\lambda^{2}$ дает голоморфную функцию от $q^{2}$. Не определяемые нами ряды по $q^{2}$ мы будем обозначать как $P\left(q^{2}\right), Q\left(q^{2}\right)$. Их коэффишиенты определяются поведением функции $Z_{R}(x)$ при всех значениях аргумента, а не только инфракрасной областью. Эти ряды мы не будем различать для различных интегралов. Подставив оценку (8) в формулу (П.12), получим

$$
J_{2}^{-1}=P\left(q^{2}\right)+\alpha_{0}\left(\frac{q^{2}}{\mu^{2}}\right)^{1-c} j_{2}^{-1}(c)+o\left(\left(q^{2} / \mu^{2}\right)^{1-c}\right) .
$$

Нелидируюшие члены в формуле (8) дают вклады в не определяемый нами ряд $P\left(q^{2}\right)$ и в нелидируюшие степенные члены формулы (П.13). При $c>1$ для функции $j_{2}^{-1}(c)$ справедливо интегральное представление

$$
j_{2}^{-1}(c)=\int_{0}^{1} d t t^{-2+c}\left[(1+t)^{1-c}\right] .
$$

При рассмотрении интегралов $J_{2}^{0,1}$ удобно сначала продифференцировать интегралы с искомым инфракрасным поведением по $q^{2}$, а затем проинтегрировать по $q^{2}$ от нуля до $q^{2}$. Используя оценку (8), в результате при $c<2$ получаем

$$
\begin{aligned}
J_{2}^{0}= & \frac{1}{q^{2}} \int_{0}^{\Lambda_{\infty}^{2}} d y Z_{R}(y)+P\left(q^{2}\right)+\alpha_{0}\left(\frac{q^{2}}{\mu^{2}}\right)^{1-c} \frac{2^{2-c}}{c-2}+o\left(\left(q^{2} / \mu^{2}\right)^{1-c}\right) \\
J_{2}^{1}= & \frac{1}{q^{4}} \int_{0}^{\Lambda_{\infty}^{2}} d y y Z_{R}(y)+\frac{1}{q^{2}} \int_{0}^{\Lambda_{\infty}^{2}} d y \frac{d Z_{R}(y)}{d y}+P\left(q^{2}\right)+ \\
& +\alpha_{0}\left(\frac{q^{2}}{\mu^{2}}\right)^{1-c} \frac{(c-1) 2^{2-c}}{(c-2)(c-3)}+o\left(\left(q^{2} / \mu^{2}\right)^{1-c}\right) .
\end{aligned}
$$

Рассмотрим интеграл $J_{3}$. Вводя параметр $\lambda^{2}<\mu^{2}$ и разлагая знаменатель в ряд по степеням $y / \mu^{2}$, получаем при $c<2$

$$
J_{3}=\frac{\mu^{2}}{q^{2}} \int_{0}^{\Lambda_{\infty}^{2}} d y \frac{Z_{R}(y)}{y+\mu^{2}}+P\left(q^{2}\right)+\alpha_{0}\left(\frac{q^{2}}{\mu^{2}}\right)^{1-c} \frac{2^{2-c}}{c-2}+o\left(\left(q^{2} / \mu^{2}\right)^{1-c}\right) .
$$


Обратимся к интегралу $J_{4}^{-1}$. Интегрируя по соответствующей области, получаем

$$
J_{4}^{-1}=P\left(q^{2}\right)+\alpha_{0}^{2}\left(\frac{q^{2}}{\mu^{2}}\right)^{2-2 c} j_{4}(c)+o\left(\left(q^{2} / \mu^{2}\right)^{2-2 c}\right) .
$$

Интегральное представление для $j_{4}(c)$ при $c>1$ имеет вид

$$
j_{4}(c)=\int_{0}^{1} d t t^{-3+2 c}(1+t)^{1-c} .
$$

Для интеграла $J_{5}$ получаем

$$
J_{5}=\frac{1}{q^{2}} P\left(q^{2}\right)+\alpha_{0}^{2}\left(\frac{q^{2}}{\mu^{2}}\right)^{2-2 c} j_{5}(c)+o\left(\left(q^{2} / \mu^{2}\right)^{2-2 c}\right),
$$

где при $c>3 / 2$

$$
j_{5}(c)=\int_{0}^{1} d t t^{-4+2 c}(1+t)^{1-c} .
$$

Обратимся, наконец, к интегралу $J_{6}$. Разлагая в ряды в соответствующей области и интегрируя, получаем

$$
J_{6}=P\left(q^{2}\right)+Z_{R}\left(q^{2}\right) Q\left(q^{2}\right)+\alpha_{0}^{2}\left(\frac{q^{2}}{\mu^{2}}\right)^{2-2 c} j_{6}(c)+o\left(\left(q^{2} / \mu^{2}\right)^{2-2 c}\right) .
$$

Для $c>1$ интегральное представление имеет вид

$$
j_{6}(c)=\int_{0}^{1} d t\left(t^{-3+2 c}-t^{-2+c}\right) \frac{(1+t)^{1-c}}{1-t} .
$$

\section{Список литературы}

[1] Cudell J.R., Ross D.A.// Nucl. Phys. 1991. V. B359. P. 247; Cudell J.R. A new solution to the Dyson - Schwinger equation for the gluon propagator / Proceedings of the $4^{\text {th }}$ Blois Workshop on Elastic \& Diffractive Scattering. La Biodola, Italy, 1991.

[2] Landshoff P.V., Nachtmann O.// Z. Phys. 1987. V. C35. P. 405; Cudell J.R., Donnachie A., Landshoff P. V. // Nucl. Phys. 1989. V. B322. P. 55.

[3] Алексеев А.И.// ТМФ.1981. Т. 48. С. 324.

[4] Васильев А.Н., Письмак Ю.М., Хонконен Ю.Р. // ТМФ. 1981. Т. 48. С. 284.

[5] Baker M., Ball J.S., Zachariasen F. // Nucl. Phys. 1981. V. B186. P. 531, 560.

[6] Delbourgo R. // J. Phys.G: Nucl. Phys. 1979. V. 5. P. 603.

[7] Mandelstam S. // Phys. Rev. 1979. V. D20. P. 3223; Pagels H. // Phys. Rev. 1977. V. D15. P. 2991; Brown N., Pennington R. // Phys. Rev. 1988. V. D38. P. 2266.

[8] Алексеев А.И.// ЯФ.1981. Т. 33. С. 516; Алексеев А.И., Арбузов Б.А., Байков В.А.// ТМФ. 1982. Т. 52. С. 187; Алексеев А.И., Еднерал В.Ф.// ЯФ. 1987. Т. 45. С. 1105.

[9] Натрошвили К.Р., Хелашвили А.А., Хмаладзе В.Ю.// ТМФ. 1985. Т. 65. С. 360; Вачнадзе Л.Г., Кикнадзе Н.А., Хелашвили А.А. // ТМФ. 1995. Т. 102. С. 47.

[10] 't Hooft G.// Phys. Scripta. 1982. V. 25. P. 133; Nair V.P., Rosenzweig C. // Phys. Rev. 1985. V. D31. P. 401; Baker M., Ball J.S., Zachariasen F. // Phys. Rep. 1991. V. 209. P. 73.

[11] Cornwall J.M.// Phys. Rev. 1982. V. D26. P. 1453; Namyslowski J.M. Gluon propagator for an arbitrary momentum: Warsaw Univ. preprint IFT/13/90. Warsaw, 1990; Kiselev V.V. // Mod. Phys. Lett. 1994. V. A9. P. 2421. 
[12] Bender C.M., Cooper F., Simmons L.M.Jr.// Phys. Rev. 1989. V. D39. P. 2343; Rochev V.E. // J.Phys.A: Math. Gen. 1993. V. 26. P. 1235.

[13] Kummer W.// Acta Phys. Austr. 1975. V. 41. P. 315.

[14] Kim S.K., Baker M. // Nucl. Phys. 1980. V. B164. P. 152.

[15] Schoenmaker W.J.// Nucl. Phys. 1982. V. B191. P. 535.

[16] Alekseev A.I.// Phys. Lett. 1995. V. B344. P. 325.

[17] Alekseev A.I. On possibility of power type infrared behaviour of the gluon propagator / Proceedings of the XVII Workshop "Problems on High Energy Physics and Field Theory". Protvino, 1994.

[18] Le Yaouanc A., Oliver L., Ono S., Pène O., Raynal J.-C.// Phys. Rev. 1985. V. D31. P. 137; Илиева Н.П., Нгуен Суан Хан, Первушин В.Н. // ЯФ. 1987. Т. 45. С. 1169.

Институт физики высоких энергий

Поступила в редакцию

E-mail: alekseev@mx.ihep.su

17.III.1995 г

\section{A.I. Alekseev \\ ON INFRARED BEHAVIOUR OF THE GLUON PROPAGATOR}

In the framework of gluodynamics the well-known truncated Schwinger-Dyson equation for the gluon propagator is considered. The general case of a power infrared behaviour with non-integer exponents is investigated. The technique of extracting the non-leading terms of the nonlinear integral equation defined only by the infrared behaviour of the propagator is developed. The characteristic equation for the exponent is obtained and the interval of its values $-1 \leq c \leq 3$ is studied. It is shown that the equation for the gluon propagator in question has no solutions with a non-integer and nonhalfinteger power infrared behaviour. 